PROCEEDINGS OF THE

AMERICAN MATHEMATICAL SOCIETY

Volume 129, Number 3, Pages 725-730

S 0002-9939(00)06021-4

Article electronically published on November 3, 2000

\title{
TRIGONOMETRIC POLYNOMIALS WITH MANY REAL ZEROS AND A LITTLEWOOD-TYPE PROBLEM
}

\author{
PETER BORWEIN AND TAMÁS ERDÉLYI
}

(Communicated by David R. Larson)

\begin{abstract}
We examine the size of a real trigonometric polynomial of degree at most $n$ having at least $k$ zeros in $K:=\mathbb{R}(\bmod 2 \pi)$ (counting multiplicities). This result is then used to give a new proof of a theorem of Littlewood concerning flatness of unimodular trigonometric polynomials. Our proof is shorter and simpler than Littlewood's. Moreover our constant is explicit in contrast to Littlewood's approach, which is indirect.
\end{abstract}

\section{INTRODUCTION}

The set of all polynomials of degree $n$ with coefficients \pm 1 will be denoted by $\mathcal{L}_{n}$. Specifically

$$
\mathcal{L}_{n}:=\left\{p: p(z)=\sum_{j=0}^{n} a_{j} z^{j}, a_{j} \in\{-1,1\}\right\} .
$$

Let $D$ denote the closed unit disk of the complex plane. Let $\partial D$ denote the unit circle of the complex plane. Littlewood made the following conjecture about $\mathcal{L}_{n}$ in the fifties.

Conjecture 1.1 (Littlewood). There are at least infinitely many values of $n \in \mathbb{N}$ for which there are polynomials $p_{n} \in \mathcal{L}_{n}$ so that

$$
C_{1}(n+1)^{1 / 2} \leq\left|p_{n}(z)\right| \leq C_{2}(n+1)^{1 / 2}
$$

for all $z \in \partial D$. Here the constants $C_{1}$ and $C_{2}$ are independent of $n$.

Since the $L_{2}(\partial D)$ norm of a polynomial from $\mathcal{L}_{n}$ is exactly $(2 \pi)^{1 / 2}(n+1)^{1 / 2}$, the constants must satisfy $C_{1} \leq 1$ and $C_{2} \geq 1$. See Problem 19 of [Li-68]. While there is much literature on this problem and its variants, this is still open. See [Saf-90] and [Bor-98]. In fact, finding polynomials that satisfy just the lower bound in Conjecture 1.1 is still open. The Rudin-Shapiro polynomials satisfy the upper bound.

There is a related conjecture of Erdős [Er-62.

Received by the editors March 2, 1999

2000 Mathematics Subject Classification. Primary 41A17.

Key words and phrases. Real trigonometric polynomials, real zeros, unimodular trigonometric polynomials, flatness.

The research of the first author was supported, in part, by NSERC of Canada. The research of the second author was supported, in part, by the NSF under Grant No. DMS-9623156.

(C)2000 Copyright retained by the authors 
Conjecture 1.2 (Erdös). There is a constant $\varepsilon>0$ (independent of $n$ ) so that

$$
\max _{z \in \partial D}\left|p_{n}(z)\right| \geq(1+\varepsilon)(n+1)^{1 / 2}
$$

for every $p_{n} \in \mathcal{L}_{n}$ and $n \in \mathbb{N}$. That is, the constant $C_{2}$ in Conjecture 1.1 must be bounded away from 1 (independently of $n$ ).

This conjecture is also open. Kahane [Kah-85], however, shows that if the polynomials are allowed to have complex coefficients of modulus 1 , then Conjecture 1.1 holds and Conjecture 1.2 fails. That is, for every $\varepsilon>0$ there are infinitely many values of $n \in \mathbb{N}$ for which there are polynomials $p_{n}$ of degree $n$ with complex coefficients of modulus 1 that satisfy

$$
(1-\varepsilon)(n+1)^{1 / 2} \leq\left|p_{n}(z)\right| \leq(1+\varepsilon)(n+1)^{1 / 2}
$$

for all $z \in \partial D$. Beck Bec-95 proves Conjecture 1.1 for polynomials whose coefficients are 400th roots of unity.

Our main result is a reproving of Conjecture 1.2 for real trigonometric polynomials. This is Corollary 2.4 of the next section. Littlewood gives a proof of this in [Li-61] and explores related issues in [Li-62], [Li-66a , and [Li-66b]. The approach is via Theorem 2.1 which estimates the measure of the set where a real trigonometric polynomial of degree at most $n$ with at least $k$ zeros in $K:=\mathbb{R}(\bmod 2 \pi)$ is small. There are two reasons for doing this. First the approach is, we believe, easier, and secondly it leads to explicit constants.

\section{NEW RESULTS}

Let $K:=\mathbb{R}(\bmod 2 \pi)$. For the sake of brevity the uniform norm of a continuous function $p$ on $K$ will be denoted by $\|p\|_{K}:=\|p\|_{L_{\infty}(K)}$. Let $\mathcal{T}_{n}$ denote the set of all real trigonometric polynomials of degree at most $n$, and let $\mathcal{T}_{n, k}$ denote the subset of those elements of $\mathcal{T}_{n}$ that have at least $k$ zeros in $K$ (counting multiplicities).

Theorem 2.1. Suppose $p \in \mathcal{T}_{n}$ has at least $k$ zeros in $K$ (counting multiplicities). Let $\alpha \in(0,1)$. Then

$$
m\left\{t \in K:|p(t)| \leq \alpha\|p\|_{K}\right\} \geq \frac{\alpha}{e} \frac{k}{n},
$$

where $m(A)$ denotes the one-dimensional Lebesgue measure of $A \subset K$.

Theorem 2.2. We have

$$
2 \pi\left(1-\frac{c_{2} k}{n}\right) \leq \sup _{p \in \mathcal{T}_{n, k}} \frac{\|p\|_{L_{1}(K)}}{\|p\|_{L_{\infty}(K)}} \leq 2 \pi\left(1-\frac{c_{1} k}{n}\right)
$$

for some absolute constants $0<c_{1}<c_{2}$.

Theorem 2.3. Assume that $p \in \mathcal{T}_{n}$ satisfies

$$
\|p\|_{L_{2}(K)} \leq A n^{1 / 2}
$$

and

$$
\left\|p^{\prime}\right\|_{L_{2}(K)} \geq B n^{3 / 2} .
$$

Then there is a constant $\varepsilon>0$ depending only on $A$ and $B$ such that

$$
\|p\|_{K}^{2} \geq(2 \pi-\varepsilon)^{-1}\|p\|_{L_{2}(K)}^{2} .
$$


Here

$$
\varepsilon=\frac{\pi^{3}}{1024 e} \frac{B^{6}}{A^{6}}
$$

works.

Corollary 2.4. Let $p \in \mathcal{T}_{n}$ be of the form

$$
p(t)=\sum_{k=1}^{n} a_{k} \cos \left(k t-\gamma_{k}\right), \quad a_{k}= \pm 1, \quad \gamma_{k} \in \mathbb{R}, \quad k=1,2, \ldots, n .
$$

Then there is a constant $\varepsilon>0$ such that

$$
\|p\|_{K}^{2} \geq(2 \pi-\varepsilon)^{-1}\|p\|_{L_{2}(K)}^{2} .
$$

Here

$$
\varepsilon:=\frac{\pi^{3}}{1024 e} \frac{1}{27}
$$

works.

\section{ProOfs}

To prove Theorem 2.1 we need the lemma below that is proved in [Bor-95, E.11 of Section 5.1 on pages 236-237].

Lemma 3.1. Let $p \in \mathcal{T}_{n}, t_{0} \in K$, and $r>0$. Then $p$ has at most enr $\left|p\left(t_{0}\right)\right|^{-1}\|p\|_{K}$ zeros in the interval $\left[t_{0}-r, t_{0}+r\right]$.

Proof of Theorem 2.1. Suppose $p \in \mathcal{T}_{n}$ has at least $k$ zeros in $K$, and let $\alpha \in(0,1)$. Then

$$
\left\{t \in K:|p(t)| \leq \alpha\|p\|_{K}\right\}
$$

can be written as the union of pairwise disjoint intervals $I_{j}, j=1,2, \ldots, m$. Each of the intervals $I_{j}$ contains a point $y_{j} \in I_{j}$ such that

$$
\left|p\left(y_{j}\right)\right|=\alpha\|p\|_{K} .
$$

Also, each zero of $p$ from $K$ is contained in one of the intervals $I_{j}$. Let $\mu_{j}$ denote the number of zeros of $p$ in $I_{j}$. Since $p \in \mathcal{T}_{n}$ has at least $k$ zeros in $K$, we have $\sum_{j=1}^{m} \mu_{j} \geq k$. Note also that Lemma 3.1 implies that

$$
\mu_{j} \leq e n\left|I_{j}\right|\left(\alpha\|p\|_{K}\right)^{-1}\|p\|_{K}=\frac{e n}{\alpha}\left|I_{j}\right| .
$$

Therefore

$$
k \leq \sum_{j=1}^{m} \mu_{j} \leq \frac{e n}{\alpha} \sum_{j=1}^{m}\left|I_{j}\right| \leq \frac{e n}{\alpha} m\left(\left\{t \in K:|p(t)| \leq \alpha\|p\|_{K}\right\}\right),
$$

and the result follows.

Proof of Theorem 2.2. The upper bound of the theorem follows from Theorem 2.1 applied with $\alpha=1 / 2$. The lower bound follows by considering

$$
p(t):=D_{m}(0)^{2}-D_{m}(k t)^{2} \in \mathcal{T}_{n, k} \quad \text { with } \quad m=\left\lfloor\frac{n}{2(k+1)}\right\rfloor,
$$


where

$$
D_{m}(t)=\frac{1}{2}+\sum_{j=1}^{m} \cos j t
$$

is the Dirichlet kernel of degree $m$.

Proof of Theorem 2.3. First note that by Bernstein's inequality for real trigonometric polynomials in $L_{2}(K)$, we have $B \leq A$. Assume that $p \in \mathcal{T}_{n}$ satisfies (2.1) and (2.2) but (2.3) does not hold with $\varepsilon=\pi$. Then

$$
M:=\|p\|_{K} \leq(2 \pi-\pi)^{-1 / 2}\|p\|_{L_{2}(K)} \leq \pi^{-1 / 2} A n^{1 / 2} .
$$

Combining this with Bernstein's inequality we obtain

$$
\left\|p^{\prime}\right\|_{K} \leq n\|p\|_{K} \leq \pi^{-1 / 2} A n^{3 / 2} .
$$

Using (2.2), we obtain

$$
\begin{aligned}
B^{2} n^{3} & \leq\left\|p^{\prime}\right\|_{L_{2}(K)}^{2}=\int_{K}\left|p^{\prime}(t)\right|^{2} d t \\
& \leq\left\|p^{\prime}\right\|_{K} \int_{K}\left|p^{\prime}(t)\right| d t \leq \pi^{-1 / 2} A n^{3 / 2}\left\|p^{\prime}\right\|_{L_{1}(K)},
\end{aligned}
$$

that is,

$$
\left\|p^{\prime}\right\|_{L_{1}(K)} \geq \pi^{1 / 2} \frac{B^{2}}{A} n^{3 / 2} .
$$

Associated with $p \in \mathcal{T}_{n}, M=\|p\|_{K}$, and $\gamma \in[0,1]$, let

$$
A_{\gamma}=A_{\gamma}(p)=\{t \in K:|p(t)| \leq(1-\gamma) M\}
$$

and

$$
B_{\gamma}=B_{\gamma}(p)=\{t \in K:|p(t)|>(1-\gamma) M\} .
$$

Since every horizontal line $y=c$ intersects the graph of $p \in \mathcal{T}_{n}$ in at most $2 n$ points with $x$ coordinates in $K$, we have

$$
\int_{B_{\gamma}}\left|p^{\prime}(t)\right| d t \leq 4 n \gamma M \leq 4 n \gamma \pi^{-1 / 2} A n^{1 / 2} \leq \frac{\pi^{1 / 2}}{2} \frac{B^{2}}{A} n^{3 / 2}
$$

if

$$
4 \gamma \pi^{-1 / 2} A \leq \frac{\pi^{1 / 2}}{2} \frac{B^{2}}{A}, \quad \text { that is, if } \quad \gamma \leq \frac{\pi}{8} \frac{B^{2}}{A^{2}} .
$$

Now (3.3)-(3.6) give

$$
\int_{A_{\gamma}}\left|p^{\prime}(t)\right| d t \geq \frac{\pi^{1 / 2}}{2} \frac{B^{2}}{A} n^{3 / 2} \quad \text { with } \quad \gamma=\frac{\pi}{8} \frac{B^{2}}{A^{2}} .
$$

From this, with the help of (3.1) we can deduce that there is a

$$
\delta \in(-(1-\gamma) M,(1-\gamma) M)
$$

such that $p-\delta$ has at least

$$
\frac{\frac{\pi^{1 / 2}}{2} \frac{B^{2}}{A} n^{3 / 2}}{2(1-\gamma) M} \geq \frac{\pi^{1 / 2}}{4} \frac{B^{2}}{A} \frac{n^{3 / 2}}{M} \geq \frac{\pi^{1 / 2}}{4} \frac{B^{2}}{A} \frac{n^{3 / 2}}{\pi^{-1 / 2} A n^{1 / 2}}=\frac{\pi}{4} \frac{B^{2}}{A^{2}} n
$$


zeros in $K$. Therefore Theorem 2.1 yields that

$$
\begin{aligned}
m\left\{t \in K:|p(t)| \leq\left(1-\frac{\gamma}{2}\right)\|p\|_{K}\right\} & \geq m\left\{t \in K:|p(t)-\delta| \leq \frac{\gamma}{2}\|p\|_{K}\right\} \\
& \geq m\left\{t \in K:|p(t)-\delta| \leq \frac{\gamma}{4}\|p-\delta\|_{K}\right\} \\
& \geq \frac{1}{e} \frac{\gamma}{4} \frac{\pi}{4} \frac{B^{2}}{A^{2}} \frac{n}{n} \geq \frac{\pi^{2}}{128 e} \frac{B^{4}}{A^{4}} .
\end{aligned}
$$

Therefore

$$
\begin{aligned}
2 \pi\|p\|_{K}^{2}-\|p\|_{L_{2}(K)}^{2} & =\int_{K}\left(\|p\|_{K}^{2}-|p(t)|^{2}\right) d t \geq \frac{\pi^{2}}{128 e} \frac{B^{4}}{A^{4}} \frac{\gamma}{2}\|p\|_{K}^{2} \\
& =\frac{\pi^{3}}{1024 e} \frac{B^{6}}{A^{6}}\|p\|_{K}^{2} .
\end{aligned}
$$

We now conclude that

$$
\|p\|_{L_{2}(K)}^{2} \leq\left(2 \pi-\frac{\pi^{3}}{1024 e} \frac{B^{6}}{A^{6}}\right)\|p\|_{K}^{2},
$$

and the result follows.

Proof of Corollary 2.4. Let $p \in \mathcal{T}_{n}$ be of the given form. We have

$$
\|p\|_{L_{2}(K)}^{2}=\pi \sum_{k=1}^{n} a_{k}^{2}=\pi n
$$

that is,

$$
\|p\|_{L_{2}(K)}=\pi^{1 / 2} n^{1 / 2}
$$

Also

$$
\left\|p^{\prime}\right\|_{L_{2}(K)}^{2}=\pi \sum_{k=1}^{n} k^{2} a_{k}^{2}=\pi \frac{n(n+1)(2 n+1)}{6} \geq \frac{\pi}{3} n^{3}
$$

that is,

$$
\left\|p^{\prime}\right\|_{L_{2}(K)} \geq\left(\frac{\pi}{3}\right)^{1 / 2} n^{3 / 2}
$$

Now the result follows from Theorem 2.3 with $A:=\pi^{1 / 2}$ and $B:=(\pi / 3)^{1 / 2}$.

\section{REFERENCES}

[Bec-95] J. Beck, Flat polynomials on the unit circle - note on a problem of Littlewood, Bull. London Math. Soc. 23 (1991), 269-277. MR 93b:42002

[Bor-98] P. Borwein, Some Old Problems on Polynomials with Integer Coefficients, in Approximation Theory IX, ed. C. Chui and L. Schumaker, Vanderbilt University Press (1998), 31-50. CMP 2000:11

[Bor-95] P. Borwein and T. Erdélyi, Polynomials and Polynomial Inequalities, Springer-Verlag, New York, 1995. MR 97e:41001

[Er-62] P. Erdős, An inequality for the maximum of trigonometric polynomials, Annales Polonica Math. 12 (1962), 151-154. MR 25:5330

[Kah-85] J-P. Kahane, Sur les polynômes á coefficients unimodulaires, Bull. London Math. Soc 12 (1980), 321-342. MR 82a:30003

[Li-61] J.E. Littlewood, On the mean value of certain trigonometric polynomials, Jour. London Math. Soc. 36 (1961), 307-334. IMR 25:5331a

[Li-62] J.E. Littlewood, On the mean value of certain trigonometric polynomials (II), Jour. London Math. Soc. 39 (1964), 511-552. 
[Li-66a] J.E. Littlewood, The real zeros and value distributions of real trigonometrical polynomials, Jour. London Math. Soc. 41 (1966), 336-342. MR 33:4559

[Li-66b] J.E. Littlewood, On polynomials $\sum \pm z^{m}$ and $\sum e^{\alpha_{m} i} z^{m}, z=e^{\theta i}$, Jour. London Math. Soc. 41 (1966), 367-376. MR 33:4237

[Li-68] J.E. Littlewood, Some Problems in Real and Complex Analysis, Heath Mathematical Monographs, Lexington, Massachusetts, 1968. MR 39:5777

[Saf-90] B. Saffari, Barker sequences and Littlewood's "two sided conjectures" on polynomials with \pm 1 coefficients, Séminaire d'Analyse Harmonique, 1989/90, Univ. Paris XI, Orsay, 1990, 139-151. MR 92i:11032

Department of Mathematics and Statistics, Simon Fraser University, Burnaby, British Columbia, Canada V5A 1S6

E-mail address: pborwein@cecm.sfu.ca

Department of Mathematics, Texas A\&M University, College Station, Texas 77843

E-mail address: terdelyi@math.tamu.edu 